University of Nebraska - Lincoln

DigitalCommons@University of Nebraska - Lincoln

Biological Systems Engineering: Papers and

Publications

Biological Systems Engineering

2010

\title{
Numerically Predicting Seepage Gradient Forces and Erosion: Sensitivity to Soil Hydraulic Properties
}

\author{
Garey A. Fox \\ Oklahoma State University - Main Campus, gafox2@ncsu.edu \\ Derek M. Heeren \\ University of Nebraska-Lincoln, derek.heeren@unl.edu \\ Glenn V. Wilson \\ USDA National Sedimentation Laboratory, glenn.wilson@ars.usda.gov \\ E. J. Langendoen \\ USDA-ARS National Sedimentation Laboratory, eddy.langendoen@ars.usda.gov \\ Amanda K. Fox \\ Stillwater, OK \\ See next page for additional authors
}

Follow this and additional works at: https://digitalcommons.unl.edu/biosysengfacpub

Part of the Bioresource and Agricultural Engineering Commons, Environmental Engineering Commons, and the Other Civil and Environmental Engineering Commons

Fox, Garey A.; Heeren, Derek M.; Wilson, Glenn V.; Langendoen, E. J.; Fox, Amanda K.; and Chu-Agor, Maria L., "Numerically Predicting Seepage Gradient Forces and Erosion: Sensitivity to Soil Hydraulic Properties" (2010). Biological Systems Engineering: Papers and Publications. 302.

https://digitalcommons.unl.edu/biosysengfacpub/302

This Article is brought to you for free and open access by the Biological Systems Engineering at DigitalCommons@University of Nebraska - Lincoln. It has been accepted for inclusion in Biological Systems Engineering: Papers and Publications by an authorized administrator of DigitalCommons@University of Nebraska Lincoln. 


\section{Authors}

Garey A. Fox, Derek M. Heeren, Glenn V. Wilson, E. J. Langendoen, Amanda K. Fox, and Maria L. Chu-Agor 


\title{
Numerically predicting seepage gradient forces and erosion: Sensitivity to soil hydraulic properties
}

\author{
Garey A. Fox ${ }^{\mathrm{a}, *}$, Derek M. Heeren ${ }^{\mathrm{b}}$, Glenn V. Wilson ${ }^{\mathrm{c}}$, Eddy J. Langendoen ${ }^{\mathrm{c}}$, Amanda K. Fox ${ }^{\mathrm{d}, 1}$, \\ Maria L. Chu-Agor ${ }^{\mathrm{e}}$ \\ ${ }^{a}$ Environmental and Natural Resources Engineering, Department of Biosystems and Agricultural Engineering, Oklahoma State University, 120 Agricultural Hall, Stillwater, OK \\ 74078, United States \\ ${ }^{\mathrm{b}}$ Department of Biosystems and Agricultural Engineering, Oklahoma State University, Stillwater, OK 74078, United States \\ ${ }^{\mathrm{C}}$ USDA-ARS National Sedimentation Laboratory, Oxford, MS, United States \\ ${ }^{\mathrm{d}}$ Stillwater, OK, United States \\ ${ }^{\mathrm{e}}$ University of Florida, Gainesville, FL, United States
}

\section{A R T I C L E I N F O}

\section{Article history:}

Received 14 October 2009

Received in revised form 12 February 2010

Accepted 7 June 2010

This manuscript was handled by Philippe Baveye, Editor-in-Chief, with the assistance of D.P. Ahlfeld, Associate Editor

\section{Keywords:}

Groundwater seepage

Pedotransfer function

ROSETTA

Seepage erosion

Uncertainty analysis

\begin{abstract}
S U M M A R Y
Research has suggested that streambank seepage can be an important mechanism of bank instability; however, limited information is available on the level of soil characterization necessary to accurately predict seepage gradient forces and erosion. The objective of this research was to quantify the expected range of predicted seepage gradients for various degrees of site characterization. Uncertainty analysis on seepage gradient predictions was performed relative to variability in soil hydraulic properties. A two-dimensional unsaturated/saturated groundwater flow model was used to simulate a homogeneous soil layer for sand and loamy sand soils packed at various bulk densities, $\rho_{b}$. A pedotransfer function (ROSETTA), designed to estimate unsaturated hydraulic properties from surrogate soil data (i.e., texture and bulk density), was used to derive the saturated hydraulic conductivity, $K_{s}$, and water retention parameters for various levels of site information (i.e., only textural class; percent sand, silt, and clay (\%SSC); \%SSC and $\rho_{b}$; and \%SSC, $\rho_{b}$, and $K_{s}$ ). Statistical distributions were derived for each soil hydraulic parameter and Monte Carlo simulations were performed to generate distributions of maximum seepage gradient. The deviation in predicted seepage gradient was calculated using assumed baseline conditions. Ranges in predicted soil hydraulic parameters and maximum seepage gradients were considerably reduced when using \%SSC as compared to soil texture. Therefore, at a minimum, soil samples should be taken for particle size analysis. For $\rho_{b}$ between 1450 and $1500 \mathrm{~kg} \mathrm{~m}^{-3}$, soil hydraulic parameters could be derived using ROSETTA and inputting \%SSC, with little additional benefit provided by measuring $\rho_{b}$ and/or $K_{s}$. When the $\rho_{b}$ was less than $1450-1500 \mathrm{~kg} \mathrm{~m}^{-3}$, inputting $\rho_{b}$ and/or $K_{s}$ consistently reduced the magnitude of deviations from the baseline and therefore should be measured from undisturbed soil samples. The opposite was observed for $\rho_{b}$ greater than $1450-1500 \mathrm{~kg} \mathrm{~m}^{-3}$ due to discrepancies between ROSETTA-derived and actual values of soil hydraulic parameters other than $K_{s}$. Considerable deviations (i.e., around 20\%) were observed in seepage gradients under this scenario. When ROSETTA-derived and actual values of soil hydraulic parameters more closely matched, inputting $\rho_{b}$ and/or $K_{s}$ benefitted seepage gradient predictions as deviations in seepage gradients were less than $5 \%$ for the sand and loamy sand soils. Therefore, it is vital to quantify all soil hydraulic parameters for high $\rho_{b}$ soils and textures with a wide range in \%SSC.
\end{abstract}

(c) 2010 Elsevier B.V. All rights reserved.

\section{Introduction}

Streambank failures result in loss of land, increased stream sediment loads, and increased nutrient loads if soil nutrient levels are high. Long-term data from Goodwin Creek, Mississippi, show that up to $85 \%$ of the sediment leaving the catchment

\footnotetext{
* Corresponding author. Tel.: +1 4057448423.

E-mail address: garey.fox@okstate.edu (G.A. Fox).

1 Independent Consultant.
}

emanated not from the adjacent fields (cropland constituted around $10 \%$ of the area) but from the streambanks and beds (Grissinger et al., 1991; Simon and Darby, 1997; Wilson et al., 2007). Other research has also demonstrated that streambank erosion contributes significantly to total sediment loading in streams (Bull and Kirkby, 1997; Simon and Darby, 1999; Sekely et al., 2002; Evans et al., 2006). However, the relative importance of various mechanisms of streambank erosion remains unclear (Hooke, 1979; Odgaard, 1991; Fox et al., 2007a,b; Fox and Wilson, 2010). 
At least three groundwater mechanisms of streambank erosion and failure have been observed (Fox and Wilson, 2010). First, an increase in soil moisture can reduce cohesion and soil frictional strength (Darby and Thorne, 1996; Crosta and di Prisco, 1999; Rinaldi and Casagli, 1999; Simon et al., 1999). The importance of this instability mechanism has led to work by Darby et al. (2007), Rinaldi et al. (2008), and Langendoen et al. (2009) in linking groundwater flow, fluvial hydraulics, and streambank stability models.

The second and third mechanisms relate to seepage flow through soil layers towards the stream. Hagerty (1991a,b) discussed the difficulty of identifying sites with seepage in the field, especially since seepage is often intermittent. It is largely unknown whether sites with potential for seepage erosion can be predicted during a field reconnaissance based on easily defined soil properties (e.g., texture). Seepage often results from a perched water table above a restrictive layer. Wilson et al. (2007) observed a two and a half order of magnitude difference in vertical saturated hydraulic conductivity $\left(K_{s}\right)$ in a bank profile that resulted in a perched water table and seepage. This difference was the result of only a $16 \%$ change in clay content between layers. Wilson et al. (2007) also noted that such layers may be thin alluvial deposits of $5-10 \mathrm{~cm}$. Fox et al. (2007a) noted much smaller differences (around one order of magnitude) in vertical $K_{s}$ among soil layers that also resulted in seepage.

When seepage occurs, seepage gradient forces $(S F)$ exert body forces on streambank sediment proportional to the hydraulic gradient (Lobkovsky et al., 2004; Chu-Agor et al., 2009):

$S F=\gamma_{w} i V$

where $\gamma_{w}$ is the unit weight of water, $i$ is the seepage gradient, and $V$ is the volume of the soil mass. Also, undercutting due to seepage exfiltration can add to streambank instability (Fox et al., 2006; Wilson et al., 2007; Cancienne et al., 2008; Chu-Agor et al., 2008a,b; Lindow et al., 2009). At the point where water exits the bank, erosion can occur producing cave-like features called seepage undercuts (Chu-Agor et al., 2008a, 2009). As these undercuts become larger, supporting material is lost which can lead to cantilever failures. This process has been noted to occur in numerous geographical locations (Fox et al., 2007a,b; Wilson et al., 2007) and its importance on stability has been highlighted in recent research (Cancienne et al., 2008; Chu-Agor et al., 2008a,b). Empirical models have recently been proposed that relate the seepage erosion rate to the ground water flow gradient and mimic excess shear stress equations used for fluvial erosion:

$E_{r s}=k_{s e}\left[i-i_{c r}\right]^{a}$

where $E_{r s}$ is the seepage erosion rate (i.e., mass of sediment per bank face area of the undercut per time, $\left.\mathrm{kg} \mathrm{m}^{-2} \mathrm{~s}^{-1}\right), k_{s e}$ is the seepage erosion erodibility coefficient $\left(\mathrm{kg} \mathrm{m}^{-2} \mathrm{~s}^{-1}\right), a$ is a power term typically assumed to be unity (Fox et al. (2007a) reports $a=1$ for field measurements and Chu-Agor et al. (2009) reports $a=1.2$ based on laboratory experiments), $i$ is the hydraulic gradient based on the groundwater steady-state velocity, and $i_{c r}$ is the critical gradient, which was estimated by Chu-Agor et al. (2009) based on laboratory experiments for sand and loamy sand soils. Eq. (2) was based on average hydraulic gradients (i.e., based on two or fewer measurements of the water table elevation along a transect in the near-bank ground water) to simplify application of the function to field scenarios (Fox and Wilson, 2010).

For both seepage gradient forces and erosion, it is the maximum seepage gradient $\left(i_{\max }\right)$, which typically occurs near the outflow boundary (i.e., streambank face), that leads to the maximum seepage force in Eq. (1) and the maximum potential for seepage erosion at the bank face. Predicting $i_{\max }$ requires the simulation of the ground water flow dynamics in the streambank. Variably saturated flow models integrated with bank stability models are used to predict bank failure (Chu-Agor et al., 2008a,b); however, an improved understanding of the soil conditions under which seepage mechanisms become prevalent is needed (Langendoen et al., 2008). Groundwater seepage is driven by several soil hydraulic properties. A critical issue facing ground water flow models has always been estimation of the representative $K_{s}$ due to the multitude of factors that influence $K_{s}$ (Masrouri et al., 2008). This parameter has been suggested to vary more than any other engineering soil parameter (Cedergren, 1967), ranging over nine orders of magnitude for different materials. Ideally, data on water retention and hydraulic conductivity functions, among other soil properties, are needed to adequately model seepage. However, these data often are not available and are uneconomical and/or laborious to acquire.

While measured hydraulic parameters are ideal for modeling unsaturated flow, pedotransfer functions (PTFs) are a convenient option for estimating missing parameters based on readily available soil data (Nemes and Rawls, 2004). The use of one such computer program, ROSETTA, is highlighted in this paper. ROSETTA is a neural network model that predicts hydraulic parameters (residual water content $\left(\theta_{r}\right)$, saturated water content $\left(\theta_{s}\right)$, van Genuchten (1980) parameters $\left(\alpha\right.$ and $n$ ), and $K_{s}$ ) for various levels of soil data (Schaap et al., 2001). ROSETTA is composed of five hierarchical PTFs for five levels of input data: (1) soil texture only; (2) percent sand, silt, and clay (\%SSC); (3) \%SSC and bulk density $\left(\rho_{b}\right)$; and the previous parameters with water pressure at (4) field capacity and (5) at wilting point. In many cases, soil texture may be the only soil physical description available for a particular site.

The objective of this research was to quantify the expected range of predicted seepage gradients given various levels of site characterization including known, or measured, data compared to assumed values. This paper addresses seeps through a sand or loamy sand conductive layer that did not involve preferential flow through a biological or structural channel. Seeps due to preferential flow have been observed along streambanks (i.e., Fox et al., 2007a; Fox and Wilson, 2010) but were outside the scope of this investigation. This research utilized soil hydraulic parameters measured from a set of laboratory experiments on two soils susceptible to seepage (sand and loamy sand) and packed at three different $\rho_{b}$ (Chu-Agor et al., 2008b).

\section{Materials and methods}

\subsection{Simulating seepage}

A homogenous, single-layer profile $10-\mathrm{cm}$ high and $100-\mathrm{cm}$ long with a $0 \%$ slope was numerically modeled for unsaturated and saturated flow (Fig. 1). The dimensions of the layer, specifically the $10-\mathrm{cm}$ seepage layer height, were selected so as to mimic natural seepage layers observed in field experiments (Fox et al., 2006; Wilson et al., 2007). A MATLAB program (Langendoen et al., 2008) was used to solve the two-dimensional Richard's equation:

$$
\left(\frac{\theta}{\phi} S_{s}+C\right) \frac{\partial h(x, z, t)}{\partial t}=\frac{\partial}{\partial x}\left[K(h) \frac{\partial h(x, z, t)}{\partial x}\right]+\frac{\partial}{\partial z}\left[K(h) \frac{\partial h(x, z, t)}{\partial z}\right]
$$

where $h$ is the pressure head, $K(h)$ is the hydraulic conductivity, $\theta$ is the water content, $\phi$ is the porosity, $S_{s}$ is the specific storage, $C$ is the specific moisture capacity, $x$ is the lateral dimension, $z$ is the vertical dimension, and $t$ is time. Following the approach of Celia et al. (1990), Langendoen et al. (2008) approximated Eq. (3) on a cell-centered finite difference grid using a modified Picard iteration scheme coupled with a backward Euler approximation in time. The water retention function, $\theta(h)$, was defined using the van Genuchten (1980) model, and $K(h)$ was defined using the combination of the 


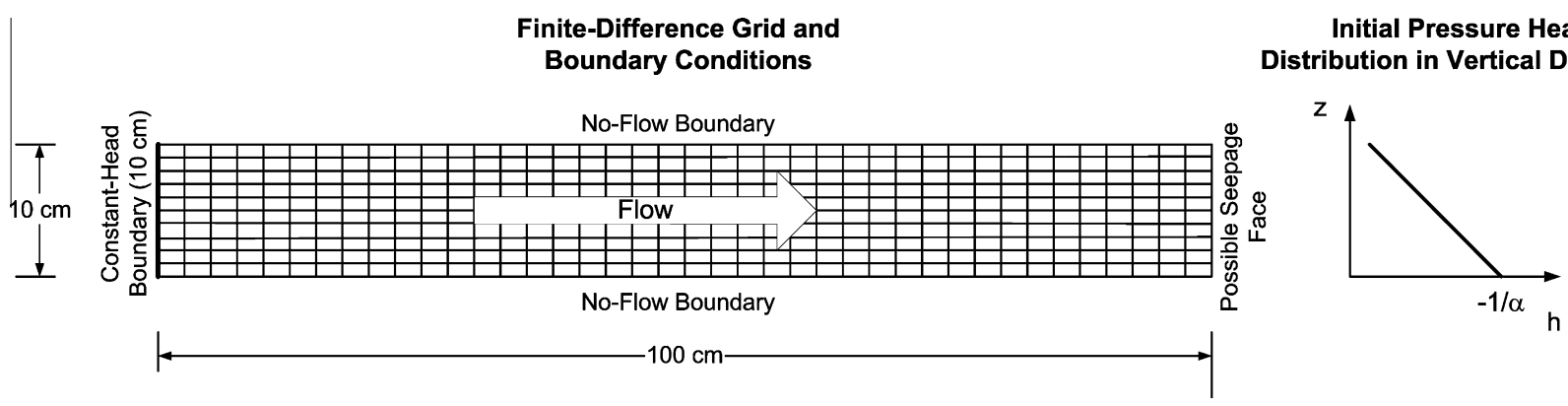

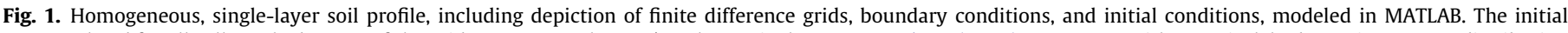

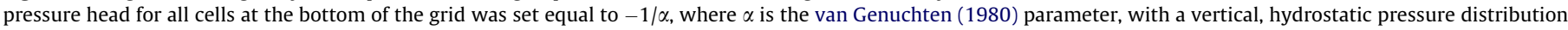
with elevation.

van Genuchten (1980) model with the Mualem (1976) pore-size distribution model:

$\theta(h)=\left\{\begin{array}{ll}\theta_{r}+\frac{\theta_{s}-\theta_{r}}{\left[1+|\alpha|^{n}\right]^{m}} & h<0 \\ \theta_{s} & h \geqslant 0\end{array}\right\}$

$K(h)=K_{s} S_{e}\left[1-\left(1-S_{e}^{1 / m}\right)^{m}\right]^{2}$

where $S_{e}$ is the effective saturation, $\alpha\left(\mathrm{L}^{-1}\right)$ and $n$ are empirical parameters, $m=1-1 / n, \theta_{s}$ is the saturated water content, and $\theta_{r}$ is the residual water content.

Boundary conditions included a zero-flux boundary for the top and bottom boundaries and a constant-head $(10 \mathrm{~cm})$ boundary condition to simulate a fixed water table (WT) on the side opposite the bank face, potentially induced by recharge to the seepage layer through infiltration or upslope ground water flow. Since seepage usually initiates following the wetting up of the bank profile (Fox et al., 2007a), initial pressure head conditions were simulated in the $10-\mathrm{cm}$ profile as near saturation (i.e., $-1 / \alpha$ ) at the bottom and hydrostatic (decreased linearly) with elevation (Fig. 1). Simulations were performed until quasi steady state flow, defined as the time when the cumulative non-zero seepage outflow per unit width of the layer, $Q^{\prime}\left(\mathrm{cm}^{2} / \mathrm{s}\right)$, deviated by no more than $10^{-6} \mathrm{~cm}^{2} / \mathrm{s}$ between time steps. The simulation time step was adjusted to minimize mass balance errors and was typically on the order of $10 \mathrm{~s}$ for each simulation.

\subsection{Seepage Erosion Uncertainty Analysis}

Chu-Agor et al. (2008b) performed seepage flow experiments with repacked soil banks at numerous $\rho_{b}$ in the laboratory. During the experiments, triplicate samples were extracted from the repacked soil block setup and were analyzed in the laboratory to determine particle size distribution and soil hydraulic properties
Table 2

Monte Carlo analysis scenarios simulating soil layers based on laboratory analysis of water retention parameters for sand and loamy sand.

\begin{tabular}{|c|c|c|}
\hline $\begin{array}{l}\text { Soil } \\
\text { type }\end{array}$ & $\begin{array}{l}\text { Scenario } \\
\text { number }\end{array}$ & Available data \\
\hline \multicolumn{3}{|l|}{ Sand } \\
\hline & 1 & Texture only \\
\hline & 2 & $\% \mathrm{SSC}^{\mathrm{a}}(99.3 \%$ sand, $0.7 \%$ silt, $0.0 \%$ clay $)$ \\
\hline & 3 & $\% \mathrm{SSC}+\rho_{b}{ }^{\mathrm{b}}=1300 \mathrm{~kg} \mathrm{~m}^{-3}$ \\
\hline & 4 & $\% \mathrm{SSC}+\rho_{b}=1300 \mathrm{~kg} \mathrm{~m}^{-3}+$ measured $K_{s}^{\mathrm{c}}$ \\
\hline & 5 & $\begin{array}{l}\text { Baseline: measured soil hydraulic parameters at } \\
\rho_{b}=1300 \mathrm{~kg} \mathrm{~m}^{-3}\end{array}$ \\
\hline & 6 & $\% \mathrm{SSC}+\rho_{b}=1450 \mathrm{~kg} \mathrm{~m}^{-3}$ \\
\hline & 7 & $\% \mathrm{SSC}+\rho_{b}=1450 \mathrm{~kg} \mathrm{~m}^{-3}+$ measured $K_{s}$ \\
\hline & 8 & $\begin{array}{l}\text { Baseline: measured soil hydraulic parameters at } \\
\rho_{b}=1450 \mathrm{~kg} \mathrm{~m}^{-3}\end{array}$ \\
\hline & 9 & $\% \mathrm{SSC}+\rho_{b}=1600 \mathrm{~kg} \mathrm{~m}^{-3}$ \\
\hline & 10 & $\% \mathrm{SSC}+\rho_{b}=1600 \mathrm{~kg} \mathrm{~m}^{-3}+$ measured $K_{s}$ \\
\hline & 11 & $\begin{array}{l}\text { Baseline: measured soil hydraulic parameters at } \\
\rho_{b}=1600 \mathrm{~kg} \mathrm{~m}^{-3}\end{array}$ \\
\hline \multicolumn{3}{|c|}{ Loamy sand } \\
\hline & 12 & Texture only \\
\hline & 13 & \%SSC $(84.5 \%$ sand, $13.4 \%$ silt, $2.1 \%$ clay) \\
\hline & 14 & $\% \mathrm{SSC}+\rho_{b}=1500 \mathrm{~kg} \mathrm{~m}^{-3}$ \\
\hline & 15 & $\% \mathrm{SSC}+\rho_{b}=1500 \mathrm{~kg} \mathrm{~m}^{-3}+$ measured $K_{s}$ \\
\hline & 16 & $\begin{array}{l}\text { Baseline: measured soil hydraulic parameters at } \\
\rho_{b}=1500 \mathrm{~kg} \mathrm{~m}^{-3}\end{array}$ \\
\hline & 17 & $\% \mathrm{SSC}+\rho_{b}=1600 \mathrm{~kg} \mathrm{~m}^{-3}$ \\
\hline & 18 & $\% \mathrm{SSC}+\rho_{b}=1600 \mathrm{~kg} \mathrm{~m}^{-3}+$ measured $K_{s}$ \\
\hline & 19 & $\begin{array}{l}\text { Baseline: measured soil hydraulic parameters at } \\
\rho_{b}=1600 \mathrm{~kg} \mathrm{~m}^{-3}\end{array}$ \\
\hline & 20 & $\% \mathrm{SSC}+\rho_{b}=1700 \mathrm{~kg} \mathrm{~m}^{-3}$ \\
\hline & 21 & $\% \mathrm{SSC}+\rho_{b}=1700 \mathrm{~kg} \mathrm{~m}^{-3}+$ measured $K_{s}$ \\
\hline & 22 & $\begin{array}{l}\text { Baseline: measured soil hydraulic parameters at } \\
\rho_{b}=1700 \mathrm{~kg} \mathrm{~m}^{-3}\end{array}$ \\
\hline
\end{tabular}

a $\%$ SSC = Percent sand, silt, and clay.

b $\rho_{b}=$ Bulk density.

c $K_{s}=$ Saturated hydraulic conductivity.

Table 1

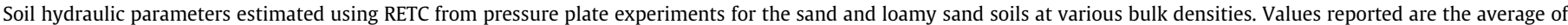
triplicate replications. Data originally reported by Chu-Agor et al. (2008b).

\begin{tabular}{|c|c|c|c|c|c|c|c|}
\hline \multirow[t]{2}{*}{ Soil type } & \multirow[t]{2}{*}{ Bulk density, $\rho_{b}\left(\mathrm{~kg} \mathrm{~m}^{-3}\right)$} & \multicolumn{6}{|c|}{ Soil hydraulic parameters ${ }^{\mathrm{a}}$} \\
\hline & & $\theta_{r}\left(\mathrm{~cm}^{3} \mathrm{~cm}^{-3}\right)$ & $\theta_{s}\left(\mathrm{~cm}^{3} \mathrm{~cm}^{-3}\right)$ & $\alpha\left(\mathrm{cm}^{-1}\right)$ & $n$ & $R^{2}$ for water retention curve fit & $K_{s}\left(\mathrm{~cm} \mathrm{~s}^{-1}\right)$ \\
\hline \multicolumn{8}{|l|}{ Sand $^{\mathrm{b}}$} \\
\hline & 1300 & 0.06 & 0.51 & 0.048 & 1.22 & 0.92 & 0.028 \\
\hline & 1450 & 0.05 & 0.46 & 0.026 & 1.28 & 0.96 & 0.017 \\
\hline & 1600 & 0.05 & 0.40 & 0.031 & 1.33 & 0.94 & 0.008 \\
\hline \multicolumn{8}{|c|}{ Loamy sand ${ }^{\mathrm{b}}$} \\
\hline & 1500 & 0.04 & 0.43 & 0.017 & 1.27 & 0.90 & 0.003 \\
\hline & 1600 & 0.04 & 0.40 & 0.026 & 1.23 & 0.94 & 0.001 \\
\hline & 1700 & 0.06 & 0.36 & 0.019 & 1.33 & 0.96 & 0.001 \\
\hline
\end{tabular}

\footnotetext{
a $\theta_{r}, \theta_{s}=$ Residual and saturated water content; $\alpha, n=$ van Genuchten (1980) parameters; $K_{s}=$ saturated hydraulic conductivity.
}

b Sand: $99.3 \%$ sand, $0.7 \%$ silt, $0.0 \%$ clay; Loamy Sand: $84.5 \%$ sand, $13.4 \%$ silt, and $2.1 \%$ clay. 
Table 3

Recommended statistical distributions and associated parameters (Meyer et al., 1997) for soil hydraulic parameters relative to soil type.

\begin{tabular}{|c|c|c|c|}
\hline $\begin{array}{l}\text { Soil } \\
\text { type }\end{array}$ & $\begin{array}{l}\text { Soil hydraulic } \\
\text { parameter }^{\mathrm{a}}\end{array}$ & $\begin{array}{l}\text { Distribution } \\
\text { type }\end{array}$ & Distribution parameters ${ }^{b}$ \\
\hline \multicolumn{4}{|l|}{ Sand } \\
\hline & $\theta_{r}$ & Lognormal & $\begin{array}{l}\gamma, \zeta, A=\exp (\gamma-3.09 \zeta) \\
B=\exp (\gamma+3.09 \zeta)\end{array}$ \\
\hline & $\theta_{s}$ & Normal & $\begin{array}{l}\mu, \sigma, A=\mu-3.09 \sigma \\
B=\mu+3.09 \sigma\end{array}$ \\
\hline & $\alpha$ & Normal & $\begin{array}{l}\mu, \sigma, A=\mu-3.09 \sigma \\
B=\mu+3.09 \sigma\end{array}$ \\
\hline & $n$ & Lognormal & $\begin{array}{l}\gamma, \zeta, A=\exp (\gamma-3.09 \zeta) \\
B=\exp (\gamma+3.09 \zeta)\end{array}$ \\
\hline & $K_{s}$ & Beta & $q, r, A, B$ \\
\hline \multicolumn{4}{|c|}{ Loamy sand } \\
\hline & $\theta_{r}$ & Normal & $\begin{array}{l}\mu, \sigma, A=\mu-3.09 \sigma, \\
B=\mu+3.09 \sigma\end{array}$ \\
\hline & $\theta_{\mathrm{s}}$ & Normal & $\begin{array}{l}\mu, \sigma, A=\mu-3.09 \sigma \\
B=\mu+3.09 \sigma\end{array}$ \\
\hline & $\alpha$ & Lognormal & $\begin{array}{l}\gamma, \zeta, A=\exp (\gamma-3.09 \zeta) \\
B=\exp (\gamma+3.09 \zeta)\end{array}$ \\
\hline & $n$ & Lognormal & $\begin{array}{l}\gamma, \zeta, A=\exp (\gamma-3.09 \zeta) \\
B=\exp (\gamma+3.09 \zeta)\end{array}$ \\
\hline & $K_{s}$ & Beta & $q, r, A, B$ \\
\hline
\end{tabular}

a $\theta_{r}, \theta_{s}=$ Residual and saturated water content; $\alpha, n=$ van Genuchten (1980) parameters; $K_{s}=$ saturated hydraulic conductivity.

${ }^{\mathrm{b}} \mu, \sigma=$ Average and standard deviation of the normal distribution; $\gamma$, $\zeta=$ parameters of the lognormal distribution; $q, r=$ parameters controlling the shape of the beta distribution; $A, B=$ lower and upper limits of the distributions.

( $K_{s}$ and the soil water retention curve parameters) relative to $\rho_{b}$ (Table 1).

Particle size analysis was determined by sieve analysis for particles larger than $0.075 \mathrm{~mm}$ and the hydrometer method for particles less than $0.075 \mathrm{~mm}$ (ASTM Standards D422-63). The $K_{s}$ was determined on extracted soil cores with $\rho_{b}$ of 1300,1450 , and $1600 \mathrm{~kg} \mathrm{~m}^{-3}$ for the sand and 1500,1600 , and $1700 \mathrm{~kg} \mathrm{~m}^{-3}$ for the loamy sand using a falling head permeameter (McWhorter and Sunada, 1977). Water retention was determined on the extracted soil cores using standard test methods (ASTM Standards D3152 and D2325). Water retention data were modeled with Retention Curve (RETC) with the van Genuchten equation using the Mualem assumption (van Genuchten et al., 1991). A summary of the data for each soil type and $\rho_{b}$ combination is outlined in Table 1 , including the $\mathrm{R}^{2}$ between the measured and fit $\theta(h)$ using RETC (van Genuchten et al., 1991). As expected, a decrease in $K_{s}$ and $\theta_{s}$ was observed with increased $\rho_{b}$ (Table 1 ). Note also that $n$ appeared lower than typical values reported in the literature for these soil types.
In order to simulate the availability of several different soil data sets (i.e., texture only, \%SSC, $\rho_{b}$, and $K_{s}$ ), several scenarios were simulated (Table 2). The hierarchy of soil data input included the following: (1) texture only such as the information gathered easily from a soil survey; (2) \%SSC to simulate a simple laboratory test on a disturbed sample; (3) \%SSC and $\rho_{b}$ to simulate more intense laboratory tests on both disturbed and undisturbed samples; (4)\%SSC, $\rho_{b}$, and $K_{s}$ to simulate a greater level of soil characterization on both disturbed and undisturbed samples; and (5) quantification of all soil hydraulic parameters $\left(\theta_{r}, \theta_{s}, \alpha, n\right.$, and $\left.K_{s}\right)$. Three $\rho_{b}$ were simulated for both the sand and loamy sand leading to a total of 11 scenarios per soil type (Table 2).

Monte Carlo Analysis (MCA) was utilized to account for uncertainty in the input parameters (Hill and Tiedeman, 2007), dependent on ROSETTA input, on seepage gradient predictions. The average and uncertainty values provided by ROSETTA were used to generate statistical distributions of each soil hydraulic parameter based on the recommended statistical distributions of Meyer et al. (1997) as shown in Table 3. Distributions included the normal (Eq. (6)), lognormal (Eq. (7)), and beta (Eq. (8)) distributions:

$$
\begin{aligned}
& f\left(x^{\prime}\right)=\frac{1}{\sqrt{2 \pi} \sigma} \exp \left[\frac{1}{2}\left(\frac{x^{\prime}-\mu}{\sigma}\right)^{2}\right] \\
& f\left(x^{\prime}\right)=\frac{1}{\sqrt{2 \pi} \zeta x^{\prime}} \exp \left[\frac{1}{2}\left(\frac{\ln \left(x^{\prime}\right)-\gamma}{\zeta}\right)^{2}\right] \\
& f\left(x^{\prime}\right)=\frac{1}{\beta(q, r)} \frac{\left(x^{\prime}-A\right)^{q-1}\left(B-x^{\prime}\right)^{r-1}}{(B-A)^{q+r-1}}
\end{aligned}
$$

where $x^{\prime}$ is the soil parameter, $f\left(x^{\prime}\right)$ is the probability density function, $\mu$ is the mean, $\sigma$ is the variance, $\gamma$ and $\zeta$ are parameters of the lognormal distribution (Eq. (7)), $q$ and $r$ are parameters of the beta distribution (Eq. (8)), and $A$ and $B$ are the lower and upper limits of the beta distribution. Lower and upper limits $(A$ and $B$ ) for the normal and lognormal distributions were assumed to be the 0.001 and 0.999 quantiles following Meyer et al. (1997) and calculated as $\mu \pm 3.09 \sigma$ for the normal distribution and $\exp (\gamma \pm 3.09 \zeta)$ for the lognormal distribution.

These distributions were used to generate input data for seepage layer simulations. The MCA generated distributions of steady-state $Q^{\prime}$ and maximum seepage velocity, $v_{\max }$, in the simulation domain for each scenario. The $v_{\max }$ was converted into a steady-state maximum gradient, $i_{\max }$, in the simulation domain:

$i_{\max }=\frac{v_{\max } \phi}{K_{s}}$

\begin{tabular}{|c|c|c|c|c|c|c|}
\hline \multirow[t]{2}{*}{ Soil type } & \multirow[t]{2}{*}{ Type of input data } & \multicolumn{5}{|c|}{ Soil hydraulic parameters ${ }^{a}$} \\
\hline & & $\theta_{r}\left(\mathrm{~cm} 3 \mathrm{~cm}^{-3}\right)$ & $\theta_{s}\left(\mathrm{~cm} 3 \mathrm{~cm}^{-3}\right)$ & $\log _{10}(\alpha) \log _{10}\left(\mathrm{~cm}^{-1}\right)$ & $\log _{10}(n)$ & $\log _{10}\left(K_{s}\right) \log _{10}(\mathrm{~cm} / \mathrm{d})$ \\
\hline \multicolumn{7}{|l|}{ Sand } \\
\hline & Texture only & $0.05(0.03)$ & $0.38(0.06)$ & $-1.453(0.254)$ & $0.50(0.18)$ & $2.81(0.59)$ \\
\hline & $\% \mathrm{SSC}^{\mathrm{b}}$ & $0.05(0.01)$ & $0.38(0.01)$ & $-1.457(0.066)$ & $0.63(0.03)$ & $3.12(0.10)$ \\
\hline & $\%$ SSC, $\rho_{b}{ }^{\mathrm{c}}=1300 \mathrm{~kg} \mathrm{~m}^{-3}$ & $0.05(0.01)$ & $0.45(0.01)$ & $-1.446(0.086)$ & $0.59(0.04)$ & $3.17(0.13)$ \\
\hline & $\% S S C, \rho_{b}=1450 \mathrm{~kg} \mathrm{~m}^{-3}$ & $0.05(0.01)$ & $0.40(0.01)$ & $-1.505(0.075)$ & $0.65(0.04)$ & $3.18(0.11)$ \\
\hline & $\% S S C, \rho_{b}=1600 \mathrm{~kg} \mathrm{~m}^{-3}$ & $0.05(0.01)$ & $0.35(0.01)$ & $-1.511(0.086)$ & $0.64(0.03)$ & $3.10(0.12)$ \\
\hline \multicolumn{7}{|c|}{ Loamy sand } \\
\hline & Texture only & $0.05(0.04)$ & $0.39(0.07)$ & $-1.500(0.470)$ & $0.24(0.16)$ & $2.00(0.64)$ \\
\hline & $\% \mathrm{SSC}^{\mathrm{a}}$ & $0.04(0.01)$ & $0.39(0.01)$ & $-1.356(0.063)$ & $0.31(0.02)$ & $2.23(0.11)$ \\
\hline & $\% S S C, \rho_{b}=1500 \mathrm{~kg} \mathrm{~m}^{-3}$ & $0.04(0.01)$ & $0.38(0.01)$ & $-1.376(0.067)$ & $0.33(0.02)$ & $2.33(0.12)$ \\
\hline & $\% \mathrm{SSC}, \rho_{b}=1600 \mathrm{~kg} \mathrm{~m}^{-3}$ & $0.04(0.01)$ & $0.35(0.01)$ & $-1.366(0.068)$ & $0.33(0.02)$ & $2.21(0.11)$ \\
\hline & $\% S S C, \rho_{b}=1700 \mathrm{~kg} \mathrm{~m}^{-3}$ & $0.04(0.01)$ & $0.33(0.01)$ & $-1.345(0.085)$ & $0.32(0.03)$ & $2.05(0.11)$ \\
\hline
\end{tabular}

Table 4

ROSETTA soil hydraulic parameter and uncertainty estimates (in parentheses) for the sand and loamy sand soils.

\footnotetext{
${ }^{\mathrm{a}} \theta_{r}, \theta_{s}=$ Residual and saturated water content; $\alpha, n=$ van Genuchten (1980) parameters; $K_{s}=$ saturated hydraulic conductivity

b $\%$ SSC = Percent sand, silt, and clay: Sand: $99.3 \%$ sand, $0.7 \%$ silt, $0.0 \%$ clay; Loamy Sand: $84.5 \%$ sand, $13.4 \%$ silt, and $2.1 \%$ clay.

c $\rho_{b}=$ Bulk density
} 
Table 5

Derived statistical distributions for the sand scenarios using the recommended distributions of Meyer et al. (1997) and ROSETTA-estimated parameters and uncertainty.

\begin{tabular}{|c|c|c|c|c|c|}
\hline \multirow[t]{2}{*}{ Scenario } & \multicolumn{5}{|c|}{ Soil hydraulic parameters ${ }^{a}$} \\
\hline & $\theta_{r}\left(\mathrm{~cm}^{3} \mathrm{~cm}^{-3}\right)$ & $\theta_{s}\left(\mathrm{~cm}^{3} \mathrm{~cm}^{-3}\right)$ & $\alpha\left(\mathrm{cm}^{-1}\right)$ & $n$ & $K_{s}(\mathrm{~cm} / \mathrm{s})$ \\
\hline Texture only & $\begin{array}{l}\gamma=-3.07 \\
\zeta=0.51 \\
\mathrm{~A}=0.01 \\
\mathrm{~B}=0.23\end{array}$ & $\begin{array}{l}\mu=0.38 \\
\sigma=0.06 \\
A=0.21 \\
B=0.55\end{array}$ & $\begin{array}{l}\mu=0.035 \\
\sigma=0.017 \\
A=0.001 \\
B=0.088\end{array}$ & $\begin{array}{l}\gamma=1.16 \\
\zeta=0.41 \\
A=1.10 \\
B=11.43\end{array}$ & $\begin{array}{l}q=0.433 \\
r=2.738 \\
A=0.001 \\
B=0.110\end{array}$ \\
\hline$\% \mathrm{SSC}^{\mathrm{b}}(99.3 \%$ sand, $0.7 \%$ silt, $0.0 \%$ clay) & $\begin{array}{l}\gamma=-3.00 \\
\zeta=0.11 \\
A=0.04 \\
B=0.07\end{array}$ & $\begin{array}{l}\mu=0.38 \\
\sigma=0.01 \\
A=0.35 \\
B=0.41\end{array}$ & $\begin{aligned} \mu & =0.035 \\
\sigma & =0.005 \\
A & =0.020 \\
B & =0.050\end{aligned}$ & $\begin{array}{l}\gamma=1.46 \\
\zeta=0.07 \\
A=3.44 \\
B=5.36\end{array}$ & $\begin{array}{l}q=6.151 \\
r=8.254 \\
A=0.007 \\
B=0.026\end{array}$ \\
\hline$\% \mathrm{SSC}+\rho_{b}{ }^{\mathrm{C}}=1300 \mathrm{~kg} \mathrm{~m}^{-3}$ & $\begin{array}{l}\gamma=-2.93 \\
\zeta=0.14 \\
A=0.03 \\
B=0.08\end{array}$ & $\begin{array}{l}\mu=0.45 \\
\sigma=0.01 \\
A=0.41 \\
B=0.49\end{array}$ & $\begin{aligned} \mu & =0.036 \\
\sigma & =0.007 \\
A & =0.014 \\
B & =0.058\end{aligned}$ & $\begin{array}{l}\gamma=1.36 \\
\zeta=0.10 \\
A=2.90 \\
B=5.21\end{array}$ & $\begin{array}{l}q=3.149 \\
r=6.162 \\
A=0.006 \\
B=0.0400\end{array}$ \\
\hline$\% \mathrm{SSC}+\rho_{b}=1300 \mathrm{~kg} \mathrm{~m}^{-3}+$ measured $K_{s}$ & “ & “ & “ & “ & 0.028 \\
\hline Baseline: measured soil hydraulic parameters & 0.06 & 0.51 & 0.048 & 1.22 & 0.028 \\
\hline$\% \mathrm{SSC}+\rho_{b}=1450 \mathrm{~kg} \mathrm{~m}^{-3}$ & $\begin{array}{l}\gamma=-2.92 \\
\zeta=0.14 \\
\mathrm{~A}=0.04 \\
\mathrm{~B}=0.08\end{array}$ & $\begin{array}{l}\mu=0.40 \\
\sigma=0.01 \\
A=0.37 \\
B=0.43\end{array}$ & $\begin{aligned} \mu & =0.031 \\
\sigma & =0.005 \\
A & =0.015 \\
B & =0.048\end{aligned}$ & $\begin{array}{l}\gamma=1.51 \\
\zeta=0.09 \\
\mathrm{~A}=3.39 \\
\mathrm{~B}=6.00\end{array}$ & $\begin{array}{l}q=3.299 \\
r=3.807 \\
A=0.007 \\
B=0.030\end{array}$ \\
\hline$\% \mathrm{SSC}+\rho_{b}=1450 \mathrm{~kg} \mathrm{~m}^{-3}+$ measured $K_{s}$ & “ & “ & “ & “ & 0.018 \\
\hline Baseline: measured soil hydraulic parameters & 0.05 & 0.46 & 0.026 & 1.28 & 0.018 \\
\hline$\% \mathrm{SSC}+\rho_{b}=1600 \mathrm{~kg} \mathrm{~m}^{-3}$ & $\begin{array}{l}\gamma=-2.97 \\
\zeta=0.14 \\
A=0.03 \\
B=0.08\end{array}$ & $\begin{aligned} \mu & =0.35 \\
\sigma & =0.01 \\
A & =0.33 \\
B & =0.38\end{aligned}$ & $\begin{array}{l}\mu=0.031 \\
\sigma=0.006 \\
A=0.012 \\
B=0.050\end{array}$ & $\begin{array}{l}\gamma=1.48 \\
\zeta=0.07 \\
\mathrm{~A}=3.54 \\
\mathrm{~B}=5.47\end{array}$ & $\begin{array}{l}q=3.020 \\
r=4.426 \\
A=0.006 \\
B=0.028\end{array}$ \\
\hline$\% \mathrm{SSC}+\rho_{b}=1600 \mathrm{~kg} \mathrm{~m}^{-3}+$ measured $K_{s}$ & “ & “ & “ & “ & 0.008 \\
\hline Baseline: measured soil hydraulic parameters & 0.05 & 0.40 & 0.031 & 1.33 & 0.008 \\
\hline
\end{tabular}

a $\theta_{r}, \theta_{s}=$ Residual and saturated water content; $\alpha, n=$ van Genuchten (1980) parameters; $K_{s}=$ saturated hydraulic conductivity.

b $\% \mathrm{SSC}=$ Percent sand, silt, and clay.

c $\rho_{b}=$ Bulk density.

Table 6

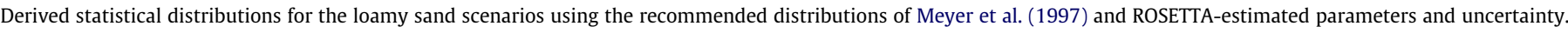

\begin{tabular}{|c|c|c|c|c|c|}
\hline \multirow[t]{2}{*}{ Scenario } & \multicolumn{5}{|c|}{ Soil hydraulic parameters ${ }^{a}$} \\
\hline & $\theta_{r}\left(\mathrm{~cm}^{3} \mathrm{~cm}^{-3}\right)$ & $\theta_{s}\left(\mathrm{~cm}^{3} \mathrm{~cm}^{-3}\right)$ & $\alpha\left(\mathrm{cm}^{-1}\right)$ & $n$ & $K_{s}(\mathrm{~cm} / \mathrm{s})$ \\
\hline Texture only & $\begin{array}{l}\mu=0.05 \\
\sigma=0.04 \\
A=0.00 \\
B=0.18\end{array}$ & $\begin{array}{l}\mu=0.39 \\
\sigma=0.07 \\
A=0.17 \\
B=0.61\end{array}$ & $\begin{array}{l}\gamma=-3.454 \\
\zeta=1.082 \\
\mathrm{~A}=0.001 \\
\mathrm{~B}=0.896\end{array}$ & $\begin{array}{l}\gamma=0.55 \\
\zeta=0.37 \\
\mathrm{~A}=1.50 \\
\mathrm{~B}=5.42\end{array}$ & $\begin{array}{l}q=0.572 \\
r=8.904 \\
A=0.000 \\
B=0.037\end{array}$ \\
\hline$\% \mathrm{SSC}^{\mathrm{b}}$ (84.5\% sand, $13.4 \%$ silt, $2.1 \%$ clay) & $\begin{array}{l}\mu=0.04 \\
\sigma=0.01 \\
A=0.02 \\
B=0.05\end{array}$ & $\begin{array}{l}\mu=0.39 \\
\sigma=0.01 \\
A=0.36 \\
B=0.41\end{array}$ & $\begin{array}{l}\gamma=-3.130 \\
\zeta=0.141 \\
A=0.028 \\
B=0.068\end{array}$ & $\begin{array}{l}\gamma=0.72 \\
\zeta=0.04 \\
\mathrm{~A}=1.82 \\
\mathrm{~B}=2.30\end{array}$ & $\begin{array}{l}q=3.412 \\
r=3.909 \\
A=0.001 \\
B=0.004\end{array}$ \\
\hline$\% \mathrm{SSC}+\rho_{b}{ }^{\mathrm{c}}=1500 \mathrm{~kg} \mathrm{~m}^{-3}$ & $\begin{array}{l}\mu=0.04 \\
\sigma=0.01 \\
A=0.02 \\
B=0.06\end{array}$ & $\begin{array}{l}\mu=0.38 \\
\sigma=0.01 \\
A=0.36 \\
B=0.40\end{array}$ & $\begin{array}{l}\gamma=-3.168 \\
\zeta=0.154 \\
A=0.026 \\
B=0.068\end{array}$ & $\begin{array}{l}\gamma=0.76 \\
\zeta=0.05 \\
\mathrm{~A}=1.84 \\
\mathrm{~B}=2.50\end{array}$ & $\begin{array}{l}q=3.382 \\
r=3.893 \\
A=0.001 \\
B=0.005\end{array}$ \\
\hline$\% \mathrm{SSC}+\rho_{b}=1500 \mathrm{~kg} \mathrm{~m}^{-3}+$ measured $K_{s}$ & “ & “ & “ & “ & 0.003 \\
\hline Baseline: measured soil hydraulic parameters & 0.04 & 0.43 & 0.017 & 1.27 & 0.003 \\
\hline$\% \mathrm{SSC}+\rho_{b}=1600 \mathrm{~kg} \mathrm{~m}^{-3}$ & $\begin{array}{l}\mu=0.04 \\
\sigma=0.01 \\
A=0.02 \\
B=0.06\end{array}$ & $\begin{array}{l}\mu=0.35 \\
\sigma=0.01 \\
A=0.33 \\
B=0.37\end{array}$ & $\begin{array}{l}\gamma=-3.145 \\
\zeta=0.157 \\
A=0.027 \\
B=0.070\end{array}$ & $\begin{array}{l}\gamma=0.76 \\
\zeta=0.05 \\
A=1.86 \\
B=2.47\end{array}$ & $\begin{array}{l}q=2.549 \\
r=4.546 \\
A=0.001 \\
B=0.004\end{array}$ \\
\hline$\% \mathrm{SSC}+\rho_{b}=1600 \mathrm{~kg} \mathrm{~m}^{-3}+$ measured $K_{s}$ & “ & “ & “ & “ & 0.001 \\
\hline Baseline: measured soil hydraulic parameters & 0.04 & 0.40 & 0.026 & 1.23 & 0.001 \\
\hline$\% \mathrm{SSC}+\rho_{b}=1700 \mathrm{~kg} \mathrm{~m}^{-3}$ & $\begin{array}{l}\mu=0.03 \\
\sigma=0.01 \\
A=0.02 \\
B=0.06\end{array}$ & $\begin{aligned} \mu & =0.33 \\
\sigma & =0.01 \\
A & =0.30 \\
B & =0.35\end{aligned}$ & $\begin{array}{l}\gamma=-3.096 \\
\zeta=0.195 \\
A=0.025 \\
B=0.083\end{array}$ & $\begin{array}{l}\gamma=0.73 \\
\zeta=0.06 \\
\mathrm{~A}=1.74 \\
\mathrm{~B}=2.47\end{array}$ & $\begin{array}{l}q=3.036 \\
r=6.201 \\
A=0.001 \\
B=0.003\end{array}$ \\
\hline$\% \mathrm{SSC}+\rho_{b}=1700 \mathrm{~kg} \mathrm{~m}^{-3}+$ measured $K_{s}$ & “ & “ & “ & “ & 0.001 \\
\hline Baseline: measured soil hydraulic parameters & 0.06 & 0.36 & 0.019 & 1.33 & 0.001 \\
\hline
\end{tabular}

a $\theta_{r}, \theta_{s}=$ Residual and saturated water content; $\alpha, n=$ van Genuchten (1980) parameters; $K_{s}=$ saturated hydraulic conductivity.

b $\%$ SSC $=$ Percent sand, silt, and clay.

c $\rho_{b}=$ Bulk density. 
Based on Eq. (1), the deviation in predicted $i_{\max }$ is proportional to the deviation in predicted seepage force. Also, assuming $a=1.0$ in Eq. (2), the deviation in predicted seepage erosion rate (i.e., $E_{r s} /$ $k_{s e}$ ) is proportional to the deviation in $i_{\max }$. The baseline $i_{\max }$ for calculating deviations was simulated using the exact data for all soil hydraulic parameters measured by Chu-Agor et al. (2008b), as shown in Table 1 . The deviation of the predicted $i_{\max }$ from the baseline $i_{\max }$ was calculated, and cumulative distribution functions (CDFs) of the deviations in $i_{\max }$ were derived for each scenario.

\section{Results and discussion}

Using the laboratory measured soil properties at the three different $\rho_{b}$ as baseline conditions allowed an evaluation of the deviations for six different soil conditions. Soil hydraulic parameters derived using ROSETTA and uncertainty estimates for the sand and loamy sand soils are shown in Table 4 . Considerable reduction
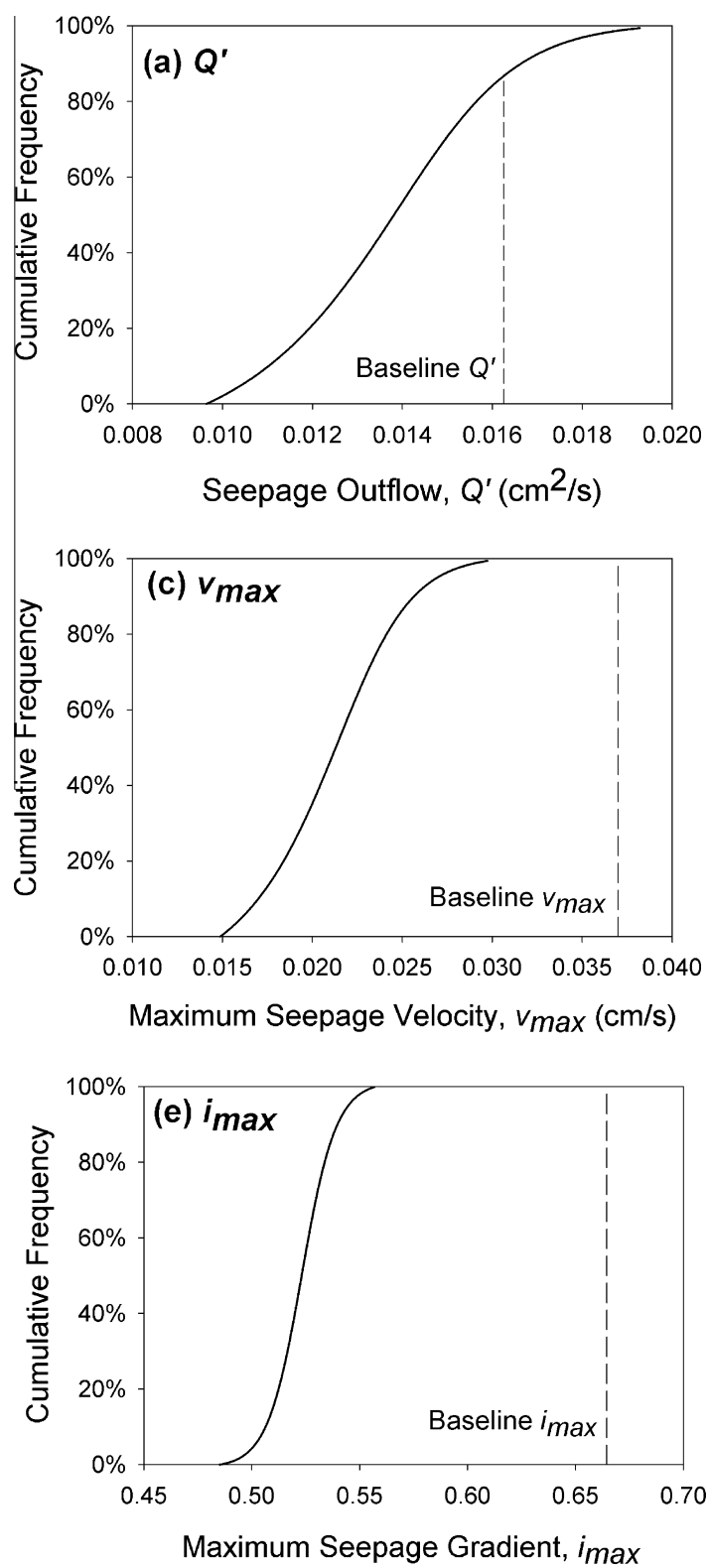

occurred in the variability of ROSETTA-derived soil hydraulic parameters when including \%SSC as opposed to only texture. Specification of the $\rho_{b}$ tended to keep the uncertainty in ROSETTA-derived parameters the same or slightly increased the uncertainty compared to only using \%SSC. These findings were most likely due to the availability of data points within the neural network training set for these soils at this selected $\rho_{b}$. Unlike ROSETTA, some PTFs which account for $\rho_{b}$ include both texture and organic matter content (Kaur et al., 2002; Nemes et al., 2005), and future research that tests these other pedotransfer functions would be valuable. Similar to the laboratory measured parameters (Table 1 ), $\theta_{s}$ and $K_{s}$ decreased with increased $\rho_{b}$, but the change in $\alpha$ was inconsistent with increased $\rho_{b}$. Also, $n$ values were consistently larger than those reported by Chu-Agor et al. (2008b). Parameters of the statistical distributions are given in Tables 5 and 6 for the sand and loamy sand soils, respectively.

The conversion between $Q^{\prime}, v_{\max }$, and $i_{\max }$ is shown in Fig. 2 for scenario 2 in Table 2 (i.e., sand soil with ROSETTA-derived soil
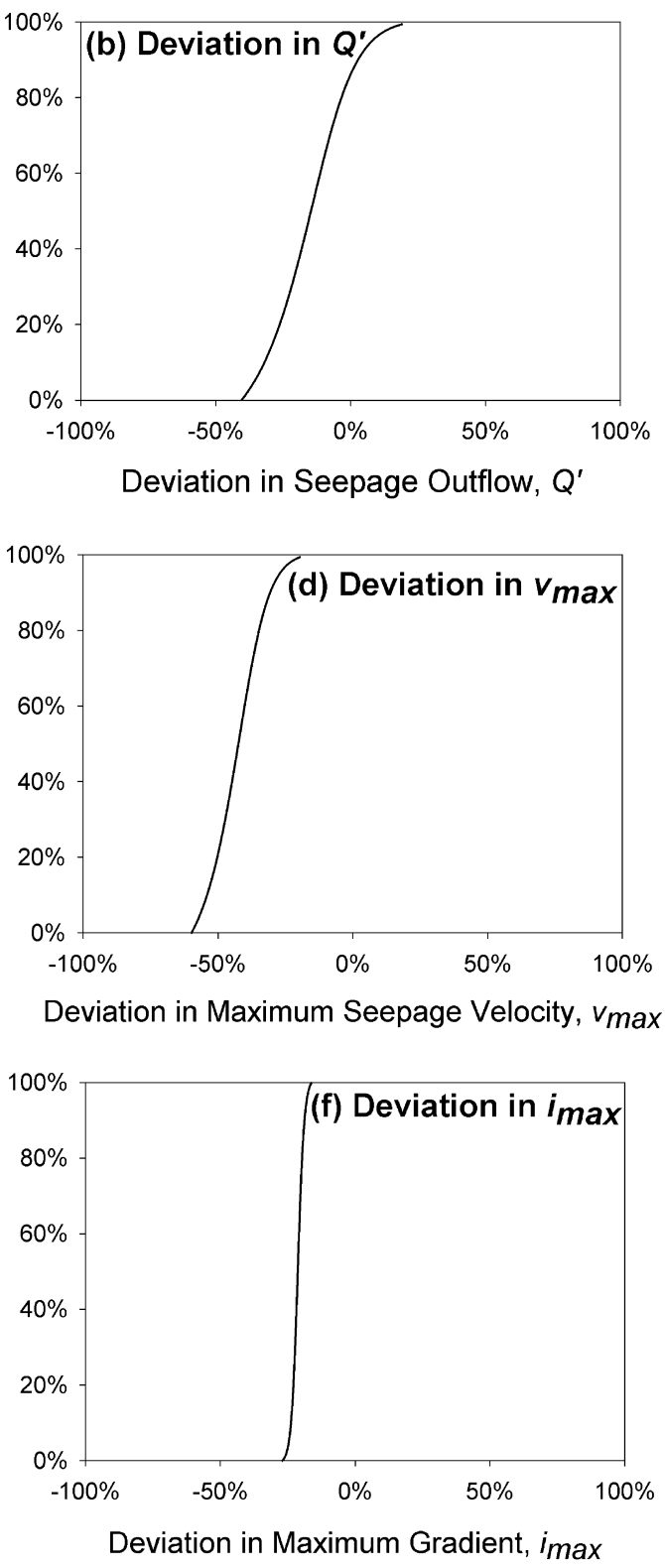

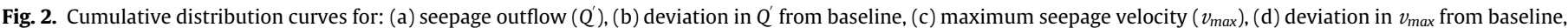

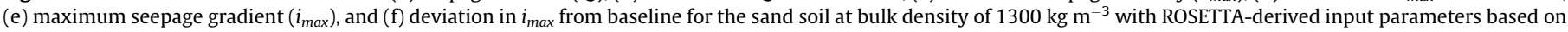
input percent sand, silt, and clay (\%SSC). 
hydraulic parameters based on \%SSC at $\rho_{b}=1300 \mathrm{~kg} \mathrm{~m}^{-3}$ ). The baseline $Q^{\prime}$ fell in the upper range of the statistical distribution because $K_{s}$ was larger for the baseline than the \%SSC- $K_{s}$ distribution (Fig. 2a and b). Also, differences between the baseline and average \%SSC ROSETTA-derived soil hydraulic parameters (most notably in the $n$ value) also resulted in a smaller perched water table depth at the outflow boundary for the baseline condition (Fig. 3). Therefore, the smaller perched water table depth at the outflow face resulted in a larger baseline $v_{\max }$ as compared to the average of the \%SSC distribution, resulting in the \%SSC $v_{\max }$ distribution falling below the baseline $v_{\max }$ (Fig. $2 \mathrm{c}$ and $\mathrm{d}$ ). As expected, the position of the baseline $i_{\max }$ relative to the \%SSC $i_{\max }$ distribution was similar (Fig. 2e and f). Deviation curves for $i_{\max }$ were much more confined than $Q^{\prime}$ and $v_{\max }$ due to the conversion based on Eq. (9) using the most variable soil hydraulic parameter, $K_{s}$. An advantage of the dependence of the erosion rate on the ground water flow gradient was the variability due to $K_{s}$ being removed from the analysis as compared to $Q^{\prime}$ or $v_{\max }$.

Knowledge of texture alone resulted in large deviations in predicted $i_{\max }$ across all simulated soil textures (Figs. 4 and 5). The $95 \%$ $\mathrm{CI}$ tended to shift towards more positive deviations (i.e., overestimation) as $\rho_{b}$ increased. For example, for the sand, the $95 \% \mathrm{CI}$ were $-46 \%$ to $-7 \%,-40 \%$ to $3 \%$, and $-30 \%$ to $18 \%$ for $\rho_{b}=1300,1450$, and $1600 \mathrm{~kg} \mathrm{~m}^{-3}$, respectively (Fig. 4). As expected, using measured \%SSC as input into ROSETTA reduced the deviation from the texture alone scenario for all six soil conditions, as shown in
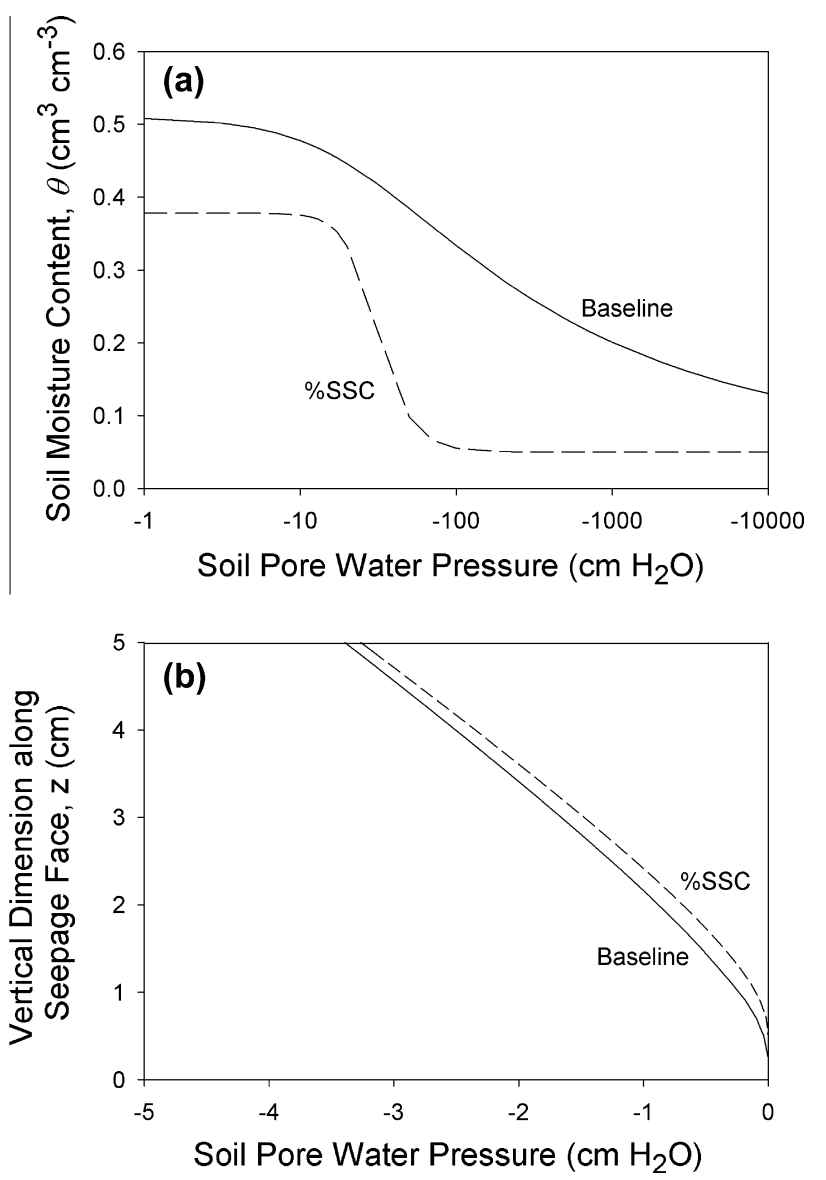

Fig. 3. (a) Water retention curves for the sand soil at bulk density of $1300 \mathrm{~kg} \mathrm{~m}^{-3}$ for the baseline condition based on laboratory measured soil hydraulic properties $\left(\theta_{r}=0.06, \theta_{s}=0.51\right.$, and $\alpha=0.048 \mathrm{~cm}^{-1}$, and $\left.n=1.3\right)$ and the ROSETTA-estimated soil hydraulic parameters based on input percent sand, silt, and clay, \%SSC $\left(\theta_{r}=0.05\right.$, $\theta_{s}=0.38$, and $\alpha=0.035 \mathrm{~cm}^{-1}$, and $n=4.3$ ). (b) Water pressure head versus elevation along the seepage face of the modeling boundary for the two different scenarios: baseline $\left(K_{s}=0.0284 \mathrm{~cm} / \mathrm{s}\right)$ and ROSETTA with $\%$ SSC $\left(K_{s}=0.0204 \mathrm{~cm} / \mathrm{s}\right)$.
Figs. 4 and 5. The 95\% CI were more confined due to the decrease in variability in ROSETTA-derived soil hydraulic parameters. The $95 \% \mathrm{CI}$ for the sand at $\rho_{b}=1300,1450$, and $1600 \mathrm{~kg} \mathrm{~m}^{-3}$ were $-24 \%$ to $-18 \%,-16 \%$ to $-9 \%$, and $-4 \%$ to $5 \%$, respectively. The $95 \%$ CI for loamy sand at $\rho_{b}=1500,1600$, and $1700 \mathrm{~kg} \mathrm{~m}^{-3}$ were $-6 \%$ to $2 \%,-5 \%$ to $3 \%$, and $-9 \%$ to $2 \%$, respectively. The $95 \%$ CI suggest that for $\rho_{b}$ at the center of sand or loamy sand soils' expected $\rho_{b}$ range, the use of \%SSC alone was a reasonable procedure for deriving soil hydraulic parameters with deviations from the baseline in $i_{\max }$ on the order of $20 \%$ or less. Therefore, knowing \%SSC reduced the deviation across both soil types. This finding suggests that at a minimum, soil samples should be taken for particle size analysis. The deviation incurred by not measuring the \%SSC directly is dependent upon the soil texture and $\rho_{b}$.

For $\rho_{b}$ in the range of $1450-1500 \mathrm{~kg} \mathrm{~m}^{-3}$, deviations in predicted $i_{\text {max }}$ when using only \%SSC were approximately equivalent to deviations in $i_{\max }$ for scenarios using $\rho_{b}$ and/or $K_{s}$ (Figs. $4 \mathrm{~b}$ and $5 a)$. When the $\rho_{b}$ for the soil was less than the $1450-1500 \mathrm{~kg} \mathrm{~m}^{-3}$ range, inputting $\rho_{b}$ and/or $K_{s}$ typically reduced the magnitude of deviations in $i_{\max }$ (Fig. 4a). For $\rho_{b}$ greater than $1450-1500 \mathrm{~kg} \mathrm{~m}^{-3}$, inputting $\rho_{b}$ and/or $K_{s}$ resulted in larger magnitude deviations in $i_{\max }$ (Figs. 4c, 5b and c), with the magnitude of the deviations increasing as $\rho_{b}$ increased.

Note that considerable deviations in $i_{\max }$ were calculated even with measurements of $\rho_{b}$ and $K_{s}$ due to significant deviations in ROSETTA-derived versus laboratory-measured values of other soil hydraulic parameters (compare Table 1 to Tables 5 and 6). More specifically, the $n$ parameter of the van Genuchten (1980) curve possessed the greatest discrepancy between ROSETTA $(n=3.2-$ 4.5 for sand, Table 5 , and $n=1.7-2.1$ for loamy sand, Table 6 ) and the laboratory measurements $(n=1.2-1.3$ for both sand and loamy sand, Tables 5 and 6). These results emphasized properly quantifying all the soil hydraulic parameters for modeling seepage through high $\rho_{b}$ soils, even if only considering steady-state seepage. Without these discrepancies in the soil hydraulic parameters other than hydraulic conductivity, deviations in seepage gradients were less than $5 \%$.

\section{Summary and conclusions}

Recent research has focused on seepage gradient forces and particle mobilization and undercutting as important bank instability mechanisms. This research investigated uncertainty in soil hydraulic parameters relative to modeling seepage gradients, a necessary variable for predicting seepage gradient forces and erosion rates. This research was applicable to seepage from homogeneous, isotropic layers of sand and loamy sand that mimicked in situ observations of streambanks. Therefore, conclusions were specific to seeps that did not involve preferential flow through biological or structural channels. Future research should consider the impact of heterogeneity and anisotropy on seepage gradient predictions, a wider range of soil types and seepage layer dimensions, and the role of preferential flow.

The advantage of the dependence of the seepage gradient force and the erosion rate on the ground water flow gradient is that some variability with respect to hydraulic conductivity is removed from the analysis as compared to seepage outflow or maximum seepage velocity, both of which depend explicitly on saturated hydraulic conductivity. Monte Carlo analyses with the various input scenarios demonstrated that knowledge of soil texture alone to derive soil hydraulic parameters for seepage gradient prediction was insufficient. Inputting percent sand, silt, and clay consistently reduced variability in soil hydraulic parameters and corresponding deviations in predicted seepage gradients from the baseline conditions. While knowledge of bulk density did not consistently reduce 

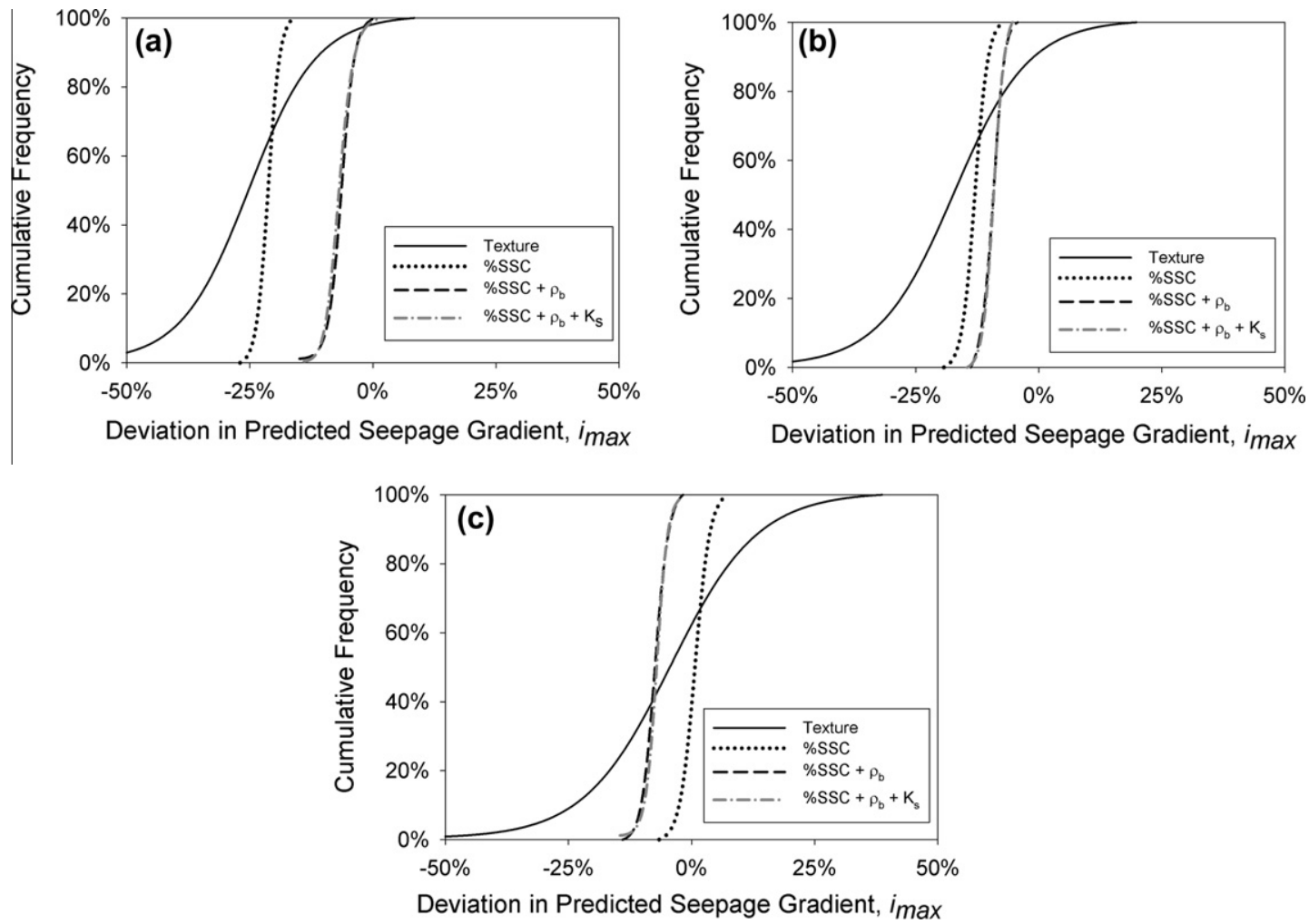

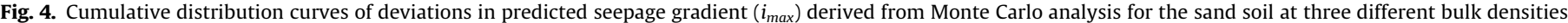

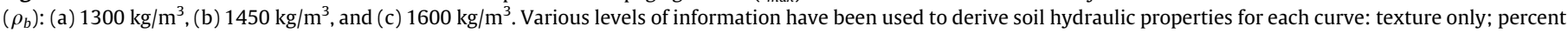
sand, silt and clay (\%SSC); \%SSC and $\rho_{b}$; and \%SSC, $\rho_{b}$, and saturated hydraulic conductivity $\left(K_{s}\right)$.
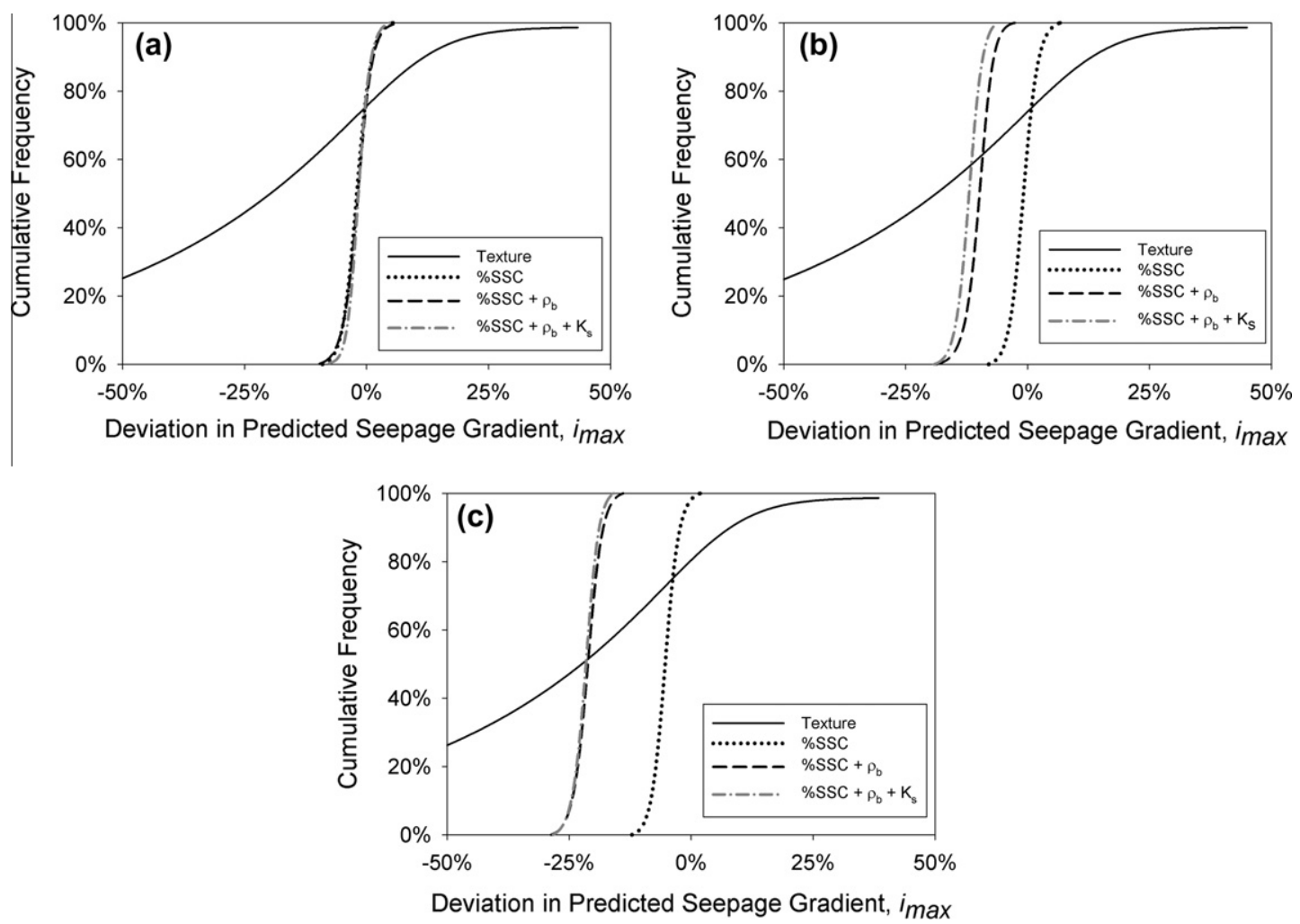

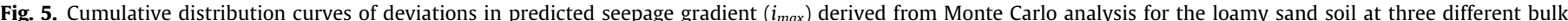

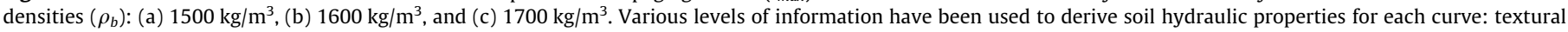
class only; percent sand, silt and clay (\%SSC); \%SSC and $\rho_{b}$; and \%SSC, $\rho_{b}$, and saturated hydraulic conductivity $\left(K_{s}\right)$. 
deviation in all scenarios, this soil parameter played a vital role in the potential range in deviations in seepage gradient predictions. For bulk densities between 1450 and $1500 \mathrm{~kg} \mathrm{~m}^{-3}$, deviations in predicted seepage gradients when using only percent sand, silt, and clay were approximately equivalent to deviations with bulk density and/or saturated hydraulic conductivity being known. When the bulk density for the soil was less than 1450$1500 \mathrm{~kg} \mathrm{~m}^{-3}$, inputting bulk density and/or saturated hydraulic conductivity consistently decreased the magnitude of deviations in seepage gradients. However, when the bulk density for the soil was greater than $1450-1500 \mathrm{~kg} \mathrm{~m}^{-3}$, inputting bulk density and/ or saturated hydraulic conductivity consistently increased the magnitude of deviations when discrepancies existed between the estimated and actual values of soil hydraulic parameters other than saturated hydraulic conductivity. More specifically, considerable deviations in seepage gradients (i.e., on the order of $20 \%$ ) can exist even with measurements of bulk density and/or saturated hydraulic conductivity when significant discrepancies exist between ROSETTA-derived versus actual values of other soil hydraulic parameters. Without these discrepancies in soil hydraulic parameters other than hydraulic conductivity, deviations in seepage gradients were generally less than $5 \%$. Therefore, for soil layers with bulk densities on the upper limits of the expected bulk density range, it is vital to have a complete quantification of the soil hydraulic parameters for accurately predicting seepage gradients.

\section{Acknowledgments}

This material is based upon work supported by the Cooperative State Research, Education, and Extension Service (CSREES), US Department of Agriculture (USDA), under Award No. 200535102-17209. The authors acknowledge three anonymous reviewers whose comments significantly improved the manuscript.

\section{References}

Bull, L.J., Kirkby, M.J., 1997. Gully processes and modelling. Progress in Physical Geography 21 (3), 354-374.

Cancienne, R., Fox, G.A., Simon, A., 2008. Influence of seepage undercutting on the root reinforcement of streambanks. Earth Surface Processes and Landforms 33 (11), 1769-1786.

Cedergren, H.R., 1967. Seepage, Drainage, and Flownets. John Wiley and Sons, Inc., New York, NY

Celia, M.A., Bouloutas, E.T., Zarba, R.L., 1990. A general mass-conservative numerical solution for the unsaturated flow equation. Water Resources Research 26 (7), 1483-1496.

Chu-Agor, M.L., Fox, G.A., Cancienne, R., Wilson, G.V., 2008a. Seepage caused tension failures and erosion undercutting of hillslopes. Journal of Hydrology 359 (3-4), 247-259. doi:10.1016/j.jhydrol.2008.07.005.

Chu-Agor, M.L., Wilson, G.V., Fox, G.A., 2008b. Numerical modeling of bank instability by seepage erosion undercutting of layered streambanks. Journal of Hydrologic Engineering 13 (12), 1133-1145.

Chu-Agor, M.L., Fox, G.A., Wilson, G.V., 2009. Empirical sediment transport function predicting seepage erosion undercutting for cohesive bank failure prediction. Journal of Hydrology 377, 155-164.

Crosta, G., di Prisco, C., 1999. On slope instability induced by seepage erosion. Canadian Journal of Geotechnical Engineering 36, 1056-1073.

Darby, S.E., Thorne, C.R., 1996. Numerical simulation of widening and bed deformation of straight sand-bed rivers. I. Model development. Journal of Hydraulic Engineering-ASCE 122, 184-193.

Darby, S.E., Rinaldi, M., Dapporto, S., 2007. Coupled simulations of fluvial erosion and mass wasting for cohesive river banks. Journal of Geophysical Research 112, F03022. doi:10.1029/2006JF000722.

Evans, D.J., Gibson, C.E., Rossell, R.S., 2006. Sediment loads and sources in heavily modified Irish catchments: a move towards informed management strategies. Geomorphology 79 (1-2), 93-113.

Fox, G.A., Wilson, G.V., 2010. The role of subsurface flow in hillslope and streambank erosion: a review. Soil Science Society of America Journal 74 (3) 717-733.

Fox, G.A., Wilson, G.V., Simon, A., Langendoen, E.J., Akay, O., Fuchs, J.W., 2007a. Measuring streambank erosion due to ground water seepage: correlation to bank pore water pressure, precipitation and stream stage. Earth Surface Processes and Landforms 32 (10), 1558-1573.
Fox, G.A., Chu-Agor, M.L., Wilson, G.V., 2007b. Seepage erosion: a significant mechanism of stream bank failure. In: Kabbes, K.C. (Ed.). Proceedings: ASCE World Environmental and Water Resources Congress, May 15-19, Tampa, FA.

Fox, G.A., Wilson, G.V., Periketi, R.K., Cullum, R.F., 2006. Sediment transport model for seepage erosion of streambank sediment. Journal of Hydrologic Engineering 11 (6), 603-611.

Grissinger, E.H., Bowie, A.J., Murphy, J.B., 1991. Goodwin Creek bank instability and sediment yield. In: Proceedings: Fifth Federal Interagency Sedimentation Conference, 32-39, March 18-21, Las Vegas, NV.

Hagerty, D.J., 1991a. Piping/sapping erosion. 1: basic considerations. Journal of Hydraulic Engineering - ASCE 117 (8), 991-1008.

Hagerty, D.J., 1991b. Piping/sapping erosion. 2: identification - diagnosis. Journal of Hydraulic Engineering - ASCE 117 (8), 1009-1025.

Hill, M.C., Tiedeman, C.R., 2007. Effective Groundwater Model Calibration: With Analysis of Data, Sensitivities, Predictions, and Uncertainties. WileyInterscience, Hoboken, NJ.

Hooke, J.M., 1979. An analysis of the processes of river bank erosion. Journal of Hydrology 42, 39-62.

Kaur, R., Kumar, S., Gurung, H.P., 2002. A pedo-transfer function for estimating soil bulk density from basic soil data and its comparison with existing PTFs. Australian Journal of Soil Research 40 (5), 847-857.

Langendoen, E.J., Lowrance, R.R., Simon, A., 2009. Assessing the impact of riparian processes on streambank stability. Ecohydrology 2 (3), 360-369.

Langendoen, E.J., Wilson, G.V., Fox, G.A., 2008. Assessing the impact of riparian soilwater dynamics on streambank erosion. In: Okay, J., Todd A. (Eds.). Riparian Ecosystems and Buffers: Working at the Water's Edge. Proceedings 2008 AWRA Summer Specialty Conference, Virginia Beach, Virginia, June 30-July 2, 2008 (CD-ROM).

Lindow, N., Fox, G.A., Evans, R.O., 2009. Seepage erosion in fluviomarine stream bank material. Earth Surface Processes and Landforms 34 (12), 1693-1701.

Lobkovsky, A.E., Jensen, B., Kudrolli, A., Rothman, D.H., 2004. Threshold phenomena in erosion driven by subsurface flow. Journal of Geophysical Research-Earth 109 (F4), F04010.

Masrouri, F., Bicalho, K.V., Kawai, K. 2008. Laboratory hydraulic testing in unsaturated soils. Geotechnical and Geological Engineering. doi: 10.1007/ s10706-008-9202-7.

McWhorter, D.B., Sunada, D.K., 1977. Ground-Water Hydrology and Hydraulics. Water Resources Publications, Highland Park, Colorado.

Meyer, P.D., Rockhold, M.L., Gee, G.W., 1997. Uncertainty Analyses of Infiltration and Subsurface Flow and Transport for SDMP Sites. US Nuclear Regulatory Commission. NUREG/CR-6565, Pacific Northwest National Laboratory.

Mualem, Y., 1976. A new model for predicting the hydraulic conductivity of unsaturated porous media. Water Resources Research 12, 513-522.

Nemes, A., Rawls, W.J., 2004. Soil texture and particle-size distribution as input to estimate soil hydraulic parameters. In: Developments in Soil Science, vol. 30. Elsevier, Amsterdam, The Netherlands. doi: 10.1016/S0166-2481(04)30004-8.

Nemes, A., Rawls, W.J., Pachepsky, Y.A., 2005. Influence of organic matter on the estimation of saturated hydraulic conductivity. Soil Science Society of America Journal 69, 1330-1337.

Odgaard, A.J., 1991. Closure to 'River meander model: I. Development'. Journal of Hydraulic Engineering - ASCE 109 (1), 142-144.

Rinaldi, M., Casagli, N., 1999. Stability of streambanks formed in partially saturated soils and effects of negative pore water pressures: the Siene River (Italy). Geomorphology 26, 253-277.

Rinaldi, M., Mengoni, B., Luppi, L., Darby, S.E., Mosselman, E., 2008. Numerical simulation of hydrodynamics and bank erosion in a river bend. Water Resources Research 44, W09428. doi:10.1029/2008WR007008.

Schaap, M.G., Leij, F.J., van Genuchten, M.Th., 2001. ROSETTA: a computer program for estimating soil hydraulic parameters with hierarchical pedotransfer functions. Journal of Hydrology 251 (3), 163-176.

Sekely, A.C., Mulla, D.J., Bauer, D.W., 2002. Streambank slumping and its contribution to the phosphorus and suspended sediment loads of the Blue Earth River, Minnesota. Journal of Soil and Water Conservation 57 (5), 243-250.

Simon, A., Darby, S.E., 1997. Disturbance, channel evolution and erosion rates: Hotophia creek, Mississippi. In: Wang S.S.Y, Langendoen, EJ. Shields, F.D. (Eds.), Proceedings: Conference on Management of Landscapes Disturbed by Channel Incision, Center for Computational Hydrosciences and Engineering, University of Mississippi, University, MS, pp. 476-481

Simon, A., Darby, S.E., 1999. The nature and significance of incised river channels In: Darby, S.E., Simon, A. (Eds.), Incised River Channels: Processes, Forms, Engineering and Management. John Wiley and Sons, Chichester, UK.

Simon, A., Curini, A., Darby, S.E., Langendoen, E.J., 1999. Streambank mechanics and the role of bank and near-bank processes in incised channels. In: Darby, S.E. Simon, A. (Eds.), Incised River Channels: Processes, Forms, Engineering and Management. John Wiley and Sons, Chichester, UK, pp. 193-217.

van Genuchten, M.Th., 1980. A closed-form equation for predicting the hydraulic conductivity of unsaturated soils. Soil Science Society of America Journal 44 (5) 892-898.

van Genuchten, M.Th., Leij, F.J., Yates, S.R., 1991. The RETC Code for Quantifying the Hydraulic Functions of Unsaturated Soils. EPA Rep. No.600/2-91/065, US Salinity Laboratory, USDA, ARS, Riverside, CA.

Wilson, G.V., Periketi, R.K., Fox, G.A., Dabney, S.M., Shields, F.D., Cullum, R.F. 2007. Soil properties controlling seepage erosion contributions to streambank failure. Earth Surface Processes and Landforms 32 (3), 447-459. 